\title{
Rare Histological Type of Adenoma of the Nonpigmented Ciliary Epithelium
}

\author{
Kentaro Ishiahara Noriyasu Hashida Kazunobu Asao Shinichi Usui \\ Kazuichi Maruyama Kohji Nishida \\ Department of Ophthalmology, Osaka University Graduate School of Medicine, \\ Osaka, Japan
}

\section{Keywords}

Ciliary tumor $\cdot$ Nonpigmented ciliary epithelium $\cdot$ Adenoma $\cdot$ Iris

\begin{abstract}
We report the rare case of an adenoma of the nonpigmented ciliary epithelium (NPCE). A 67year-old healthy man presented with a regularly shaped and nonpigmented mass at the iris root of his right eye. His best-corrected visual acuity was 1.5 with normal intraocular pressure. During observation, the size of the tumor remained stable for 1.5 years but then rapidly grew, extending through the iris, and gradually enlarged to the point of compressing the iris. Ultimately, an iridocyclectomy with scleral resection under a lamellar scleral flap was performed. The histopathologic features of the resected tissue were consistent with adenoma of the NPCE. Histopathological analysis showed that the tumor consisted of both tubular and solid components. There were solid lesions inside of the ciliary epithelium and tubular lesions outside. We observed positive immunoreactivity to vimentin and cytokeratin CK (AE1/AE3) and negative reactivity to $\mathrm{S}-100$ and $C D 68$, both rarely associated with adenoma of NPCE. During 1 year of follow-up after the iridocyclectomy, no signs of tumor recurrence were observed.
\end{abstract}




\section{Introduction}

Adenoma of the nonpigmented ciliary epithelium (NPCE) is an extremely rare benign ciliary body tumor. NPCE neoplasms have been reported to have some characteristic clinical features. Although it is known as a benign tumor, adenoma of the NPCE can cause severe local symptoms, such as inflammation [1, 2], secondary cataracts [3, 4], lens dislocation [5, 6], secondary glaucoma [5, 6], vitreous hemorrhage [2, 6], an epiretinal membrane [6], neovascularization of the optic disc, and cystoid macular edema [7]. Local resection procedures, such as iridocyclectomy, could be effective for treatment and for making a definitive diagnosis. In the current study, we report a case of an adenoma of the NPCE. Originally, the tumor did not grow. However, during a period of observation, it extended out of the iris, with an increase in size, and compressed the iris. An iridocyclectomy and cataract surgery were performed. Histopathological analysis showed that almost all of the components outside of the ciliary body were tubular components with rare histological patterns.

\section{Case Report}

A 67-year-old man who complained of a difference between his left and right iris was referred to our hospital. The patient had no prior medical history of ocular trauma. The bestcorrected visual acuity (BCVA) was 1.5 in the right eye, and the intraocular pressure was within normal limits. Slit-lamp examination of the right eye revealed an amelanotic white nodule with a regular surface that originated from the iris root (Fig. 1a). Anterior segment optical coherent tomography (AS-OCT: Casia SS-1000; Tomey, Nagoya, Japan) showed that the mass originated from the iris root and extended to the ciliary body (Fig. 1d). The left eye was normal. $N$-isopropyl-(123I)-p-iodoamphetamine ((123I)-IMP) single-photon emission computed tomography (123I-IMP SPECT) revealed no abnormal accumulation. Clinical findings indicated that the tumor was most likely not a malignant melanoma, resulting in a course of periodic observation (Fig. 1b, c, e, f). After 3 years, the visual acuity of the right eye decreased to 0.6. The mass appeared to be growing and indenting the lens, resulting in the irregular surface (Fig. 1g, j). Ultimately, iridocyclectomy was successfully performed under general anesthesia (Fig. 1h). The part of the tumor outside the iris was fragile and easily collapsed during the surgical procedures.

Histopathological examination revealed that the resected tissue was predominantly nonpigmented tumor emanating from the NPCE. The tumor was composed of tubular components and solid components. The solid components were inside of the ciliary epithelium (Fig. 2a, b), and the tubular components were outside (Fig. 2c, d). A positive reaction to vimentin and cytokeratin CK (AE1/AE3) was observed (Fig. 2a), while negative reactions to S-100 and CD68 were found in the tissue (Fig. 2d). The MIB-1 index was 8\% (Fig. 2c).

One month after the iridocyclectomy, a cataract gradually developed. The BCVA of the right eye decreased to 0.07 and cataract surgery was performed (Fig. 1i). After the surgery, the BCVA improved to 1.2. During 1 year of follow-up after iridocyclectomy and cataract surgery, no sign of tumor recurrence was seen (Fig. 1k). 


\section{Discussion}

Iris tumors are roughly divided into cystic lesions or solid lesions. Cystic lesions originally arise from the iris pigment epithelium or iris stroma. The solid tumors include melanocytic and nonmelanocytic lesions. The melanocytic iris tumors were reported to be derived from freckles, nevus (including melanocytoma), Lisch nodules, and melanoma [8]. Overall, the most common diagnoses in a clinical series of tumors include nevi, iris pigment epithelium cysts, and melanoma [8]. The nonmelanocytic iris tumors are also relatively uncommon. Adenoma of the NPCE is classified as a nonmelanocytic iris tumor [8]. In a previous analysis of 3,680 iris tumors, the frequency of adenoma of the NPCE was reported to be less than 1\% [9]. In our case, the tumor was diagnosed as an adenoma of the NPCE, a rare type of ciliary tumor. The tumor grew with extension outside the iris. The tumor bowed the iris forward and thinned the iris stroma. The size of the tumor was stable for 1.5 years. However, the tumor eventually extended through the iris into the anterior chamber with an increase in size. The resected tissue showed that most of the tumor lesions outside the iris were tubular components, whereas the lesions inside the iris were solid components. It is extremely rare that several components coexisted in this adenoma of the NPCE iris tumor.

Histopathologically, adenomas of the NPCE are usually comprised of nonpigmented cubiform or columnar cells that can assume solid, papillary, or pleomorphic patterns [10]. Most of the tumors are comprised of a variable combination of solid, papillary, and tubular components [11]. In our case, the tumor was composed of solid and tubular components, but most of the tubular components were outside the iris, while most of the solid components were inside the iris. Adenoma of the NPCE shows negative immunoreactivity for HMB-45 and positive immunoreactivity for S-100 and vimentin in most cases [6,12-15]. In some cases, a positive reaction to $\mathrm{CK}$ (AE1/AE3) is detected [1, 6, 15], CD68 is focally positive [14] or negative [15], and the proliferation index (MIB-1, Ki-67) is below $10 \%[14,15]$. In our case, a positive reaction to vimentin and CK (AE1/AE3) were seen and a negative reaction to HMB-45; however, an unusually negative reaction to $S-100$ was seen.

In the current case, negative HMB-45 immunoreactivity excludes a diagnosis of melanoma. The tumor was also composed of both tubular and solid components, and histologically negative for S-100, resulting in difficulty making a precise diagnosis. Histologically, the tumor in our case was confirmed to be benign; however, the tumor first extended through the iris and appeared in the anterior chamber by increasing its size. This is because the tubular components increased and, therefore, rapid growth of the tumor was observed.

In conclusion, we present a histologically rare case of adenoma of the NPCE comprised of solid and tubular components. During periodical observation, a malignant-like rapid growth might arise even if the composition of the tumor tissues seems to be benign. The pathogenesis still remains to be addressed, however; tubular components might be related to the rapid growth of this iris tumor.

\section{Statement of Ethics}

Written informed consent was obtained from the patient for publication of this case report and any accompanying images. A copy of the written consent is available for review by the editor of this journal. The Institutional Review Board of the Osaka University Medical School approved the research protocol, and the procedures conformed to the tenets of the Declaration of Helsinki. 
Disclosure Statement

The authors declare that there are no conflicts of interest regarding the publication of this paper.

\section{Author Contributions}

K.I., K.A., S.U., and K.M. participated in drafting the manuscript and collection, analysis, and interpretation of the data. N.H. participated in diagnosis and treatment of the patient, drafting the manuscript, and revising the manuscript. K.N. critically reviewed the manuscript and reviewed the literature. All authors consented to their names being published in this report and insist on the intellectual honesty and validity of the data provided. All authors read and approved the final version of the paper.

\section{References}

1 Bae JH, Kwon JE, Yang WI, Lee SC. Adenoma of the nonpigmented ciliary epithelium presenting with recurrent iridocyclitis: unique expression of glial fibrillary acidic protein. Graefes Arch Clin Exp Ophthalmol. 2011 Nov;249(11):1747-9.

2 Biswas J, Neelakantan A, Rao BS. Adenoma of nonpigmented epithelium of the ciliary body presenting as anterior uveitis and glaucoma: a case report. Indian J Ophthalmol. 1995 Sep;43(3):137-40.

3 Appolloni R, Modesti M, Pecorella I, Palladino D, Ciocci L. Uncommon cause of juvenile cataract: adenoma of the nonpigmented ciliary epithelium. J Cataract Refract Surg. 2008 Nov;34(11):1997-2001.

4 Argento C, Carrasco MA, Zárate JO, Zilli ML, Vilarrodona L. Ciliary body tumor and cataract: local resection combined with phacoemulsification. J Cataract Refract Surg. 2001 Jun;27(6):956-9.

5 Xian J, Xu Q, Wang Z, Yang B, Wei W, Yan Z, et al. MR imaging of adenomas of the nonpigmented ciliary epithelium of the eye. AJNR Am J Neuroradiol. 2010 May;31(5):886-90.

6 Chen ZQ, Fang XY. Adenoma of nonpigmented epithelium in ciliary body: literature review and case report. J Zhejiang Univ Sci B. 2007 Sep;8(9):612-5.

7 Suzuki J, Goto H, Usui M. Adenoma arising from nonpigmented ciliary epithelium concomitant with neovascularization of the optic disk and cystoid macular edema. Am J Ophthalmol. 2005 Jan;139(1):188-90.

8 Shields CL, Shields PW, Manalac J, Jumroendararasame C, Shields JA. Review of cystic and solid tumors of the iris. Oman J Ophthalmol. 2013 Sep;6(3):159-64.

9 Shields CL, Kancherla S, Patel J, Vijayvargiya P, Suriano MM, Kolbus E, et al. Clinical survey of 3680 iris tumors based on patient age at presentation. Ophthalmology. 2012 Feb;119(2):407-14.

10 Zimmerman LE. The remarkable polymorphism of tumors of the ciliary epithelium. Trans Aust Coll Ophthalmol. 1970;2:114-25.

11 Shields JA, Eagle RC Jr, Shields CL, De Potter P. Acquired neoplasms of the nonpigmented ciliary epithelium (adenoma and adenocarcinoma). Ophthalmology. 1996 Dec;103(12):2007-16.

12 Yan J, Liu X, Zhang P, Li Y. Acquired adenoma of the nonpigmented ciliary epithelium: analysis of five cases. Graefes Arch Clin Exp Ophthalmol. 2015 Apr;253(4):637-44.

13 Serna-Ojeda JC, Ariza-Camacho E, Collado-Solórzano A, Flores-Sánchez BC, Rodríguez-Reyes AA, FuldaGraue E. Adenoma of the Nonpigmented Ciliary Body and Iris Epithelium in Mexican Mestizo Patients. Ocul Oncol Pathol. 2015 Jun;1(4):248-53.

14 Pecorella I, Ciocci L, Modesti M, Appolloni R. Adenoma of the non-pigmented ciliary epithelium: a rare intraocular tumor with unusual immunohistochemical findings. Pathol Res Pract. 2009;205(12):870-5.

15 Sun LM, Zhang QF. Na Tang, Xue-Shan Qiu. Adenoma of the non-pigmented epithelium of the ciliary body with smooth muscle differentiation: a unique case report. Int J Clin Exp Pathol. 2016;9(11):12008-12. 


\section{Case Reports in Ophthalmology}
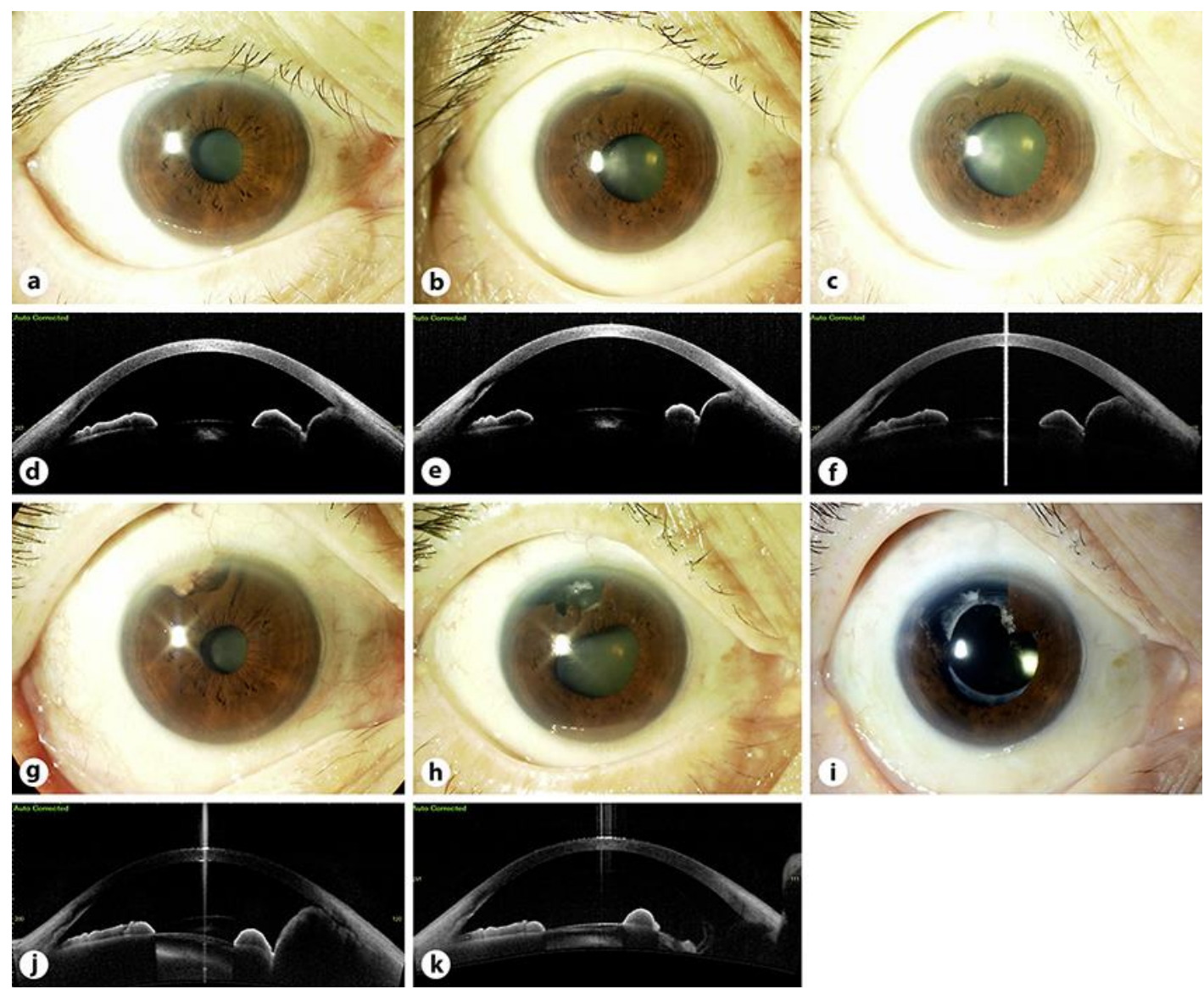

Fig. 1. A slit-lamp photograph of the right eye. a A slit-lamp photograph of the right eye shows that the mass emerged at the 11 o'clock position at the first visit. The mass grew slightly 7 months (b) and 21 months later (c). $\mathbf{d}$ An iris mass was observed on anterior segment optical coherent tomography (AS-OCT). The anterior part of the mass appeared slightly reflective (d, e) and changed to hyporeflective (f). g During the observation, the mass rapidly increased in size 24 months later. $\mathbf{h}$ Iridocyclectomy was performed. $\mathbf{i}$ One year later, cataract surgery was performed with no complication and no recurrence. $\mathbf{j}$ Due to poor visibility of the posterior surface of the iris, shadowing was observed on AS-OCT. $\mathbf{k}$ After surgery, the iris periphery could not be observed due to the surgery. 


\section{Case Reports in Ophthalmology} www.karger.com/cop

Ishiahara et al.: Rare Histological Type of Adenoma of the Nonpigmented Ciliary Epithelium

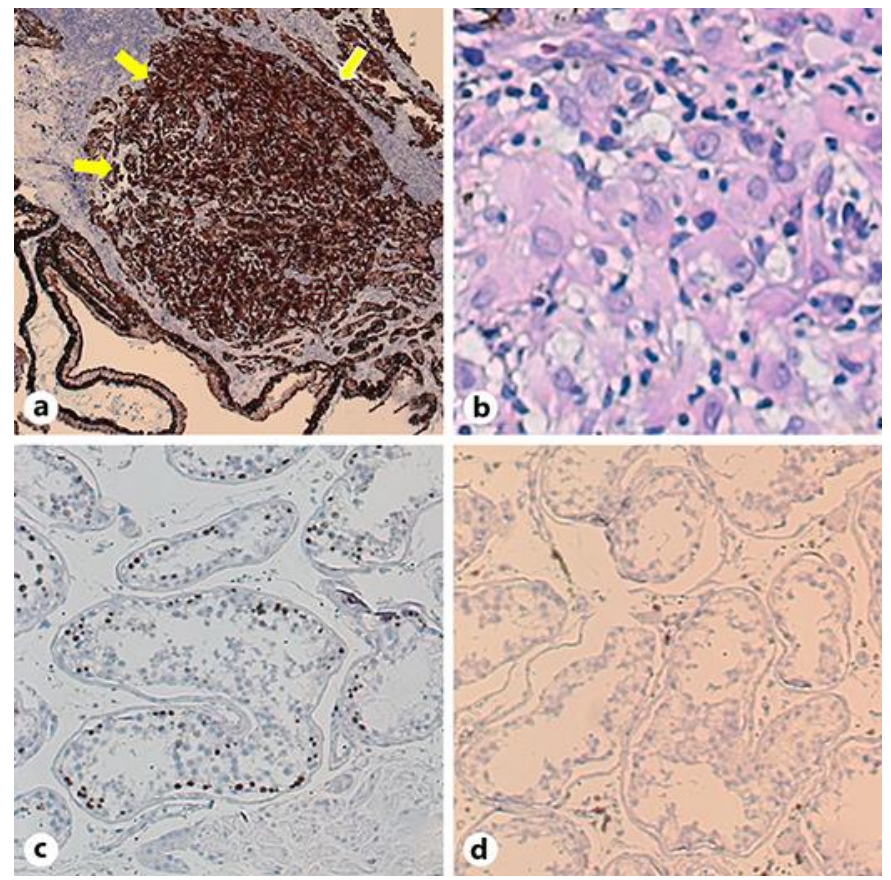

Fig. 2. Histopathology of the resected specimen inside and outside of the ciliary epithelium. a, b Histopathology of the resected specimen inside of the ciliary epithelium showed solid components. a A strong positive immunoreaction to cytokeratin AE1/AE3 can be seen (yellow arrows) inside of the ciliary epithelium. Original magnification, $\times 40$. $b$ The solid components were composed of round cells and short spindleshaped cells stained with hematoxylin and eosin. Original magnification, $\times 200$. c, $\mathbf{d}$ Histopathology of the resected specimen outside of the iris showed tubular components. c The proliferation index using MIB-1 staining was $8 \%$. Original magnification, $\times 100$. d CD68 immunostaining was negative. Original magnification, $\times 100$. 